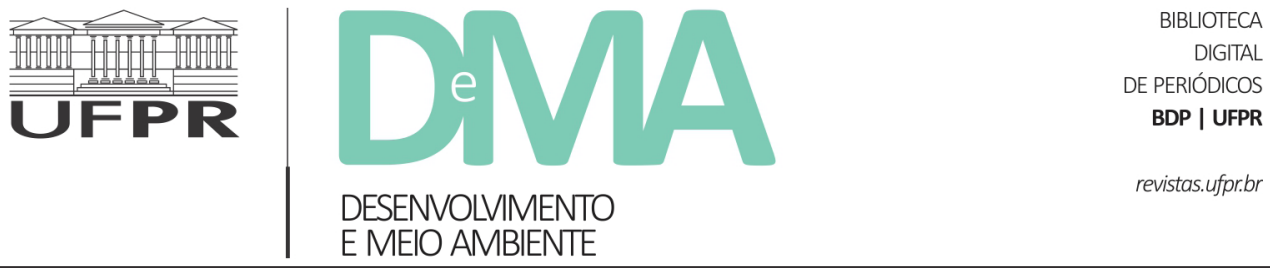

\title{
Panorama da gestão ambiental em micro e pequenas empresas amazônicas
}

\section{Panorama of environmental management in micro and small amazon companies}

\author{
Fernanda Cristina de Aguiar MENDONÇA ${ }^{1 *}$, Claudio Nahum ALVES ${ }^{1}$ \\ ${ }^{1}$ Universidade Federal do Pará (UFPA), Belém, PA, Brasil. \\ *E-mail de contato: nanda_mendonca05@hotmail.com \\ Artigo recebido em 26 de fevereiro de 2019, versão final aceita em 16 de outubro de 2020, publicado em 6 de maio de 2021.
}

RESUMO: $\quad$ Ainda que constituam 98,5\% do total de empresas no Brasil e gerem mais da metade dos empregos no país, são escassos os estudos que abordem a temática ambiental no contexto das micro e pequenas empresas. No estado do Pará, estas representam mais de $90 \%$ dos empreendimentos e geram $40 \%$ dos empregos na indústria, $68 \%$ no comércio e $43 \%$ no setor de serviços. Visando ampliar o debate acerca dessa temática, este estudo objetiva conhecer como tem se estabelecido a gestão ambiental em micro e pequenas empresas amazônicas, mais especificamente na Região Metropolitana de Belém. Para tal, uma pesquisa do tipo survey usando a Escala de Likert foi realizada. Os resultados obtidos mostraram que as práticas ecologicamente corretas mais adotadas pelas empresas da Região são as que visam redução de custos pela diminuição do uso de recursos naturais, como água e luz, e o cumprimento da legislação ambiental como forma de evitar multas e embargo de suas atividades por órgãos fiscalizadores. Ademais, os principais fatores que fazem ou fariam as empresas adotarem boas práticas estão igualmente relacionados às questões econômicas, dentre os quais: exigência do mercado consumidor, redução de custos e não pagamentos de multas ambientais. Enquanto que entre as principais barreiras encontradas para a adoção são: a falta de incentivo financeiro ou governamental, o alto custo de implantação das práticas e a falta de interesse ou cobrança por parte dos consumidores.

Palavras-chave: meio ambiente; sustentabilidade; Amazônia.

ABSTRACT: Even though they constitute $98.5 \%$ of the total number of companies in Brazil and generate more than half of the jobs in the country, there are few studies that address the environmental theme in the context of micro and small companies. In the State of Pará, these represent more than $90 \%$ of the enterprises and generate $40 \%$ of jobs in industry, $68 \%$ in commerce and $43 \%$ in the services sector. In order to broaden the debate on this topic, this study aims to understand how environmental management has been established in micro and small Amazonian companies, more specifically in the Metropolitan Region of Belém. For this purpose, a survey using the Likert Scale was carried out. The results obtained showed that the most ecologically correct 
practices adopted by companies in the Region are those aimed at reducing costs by decreasing the use of natural resources, such as water and electricity, and complying with environmental legislation as a way to avoid fines and embargoes on their activities by inspection bodies. In addition, the main factors that make or would make companies adopt good practices are also related to economic issues, among which: demand from the consumer market, cost reduction and non-payment of environmental fines. While among the main barriers found for adoption are: the lack of financial or government incentives, the high cost of implementing the practices and the lack of interest or demand from consumers.

Keywords: environment; sustainability; Amazônia.

\section{Introdução}

A preocupação com o esgotamento dos recursos naturais surgiu, após a Revolução Industrial, com a percepção da capacidade do ser humano em alterar o meio ambiente, e a constatação da existência de limites ambientais ao crescimento econômico (Seiffert, 2017). Tal fato levou a comunidade internacional, as organizações não governamentais (ONGs) e ambientalistas a pressionar governos e empresas para a elaboração de políticas que permitam a conciliação entre a atividade econômica e a proteção ambiental, visando adoção de medidas concretas na defesa do meio ambiente e na penalização do poluidor (Martins et al., 2016).

Nesse contexto, a consciência das empresas de seus impactos diretos e indiretos vem aumentando e aumentará ainda mais no futuro (Searcy, 2016). Algumas já reconhecem a importância de integrar sustentabilidade em suas políticas e atividades comerciais (Salzmann et al., 2005). Tem-se, então, a busca por padrões normativos que possibilitassem as empresas gerenciar, de forma sistematizada, ações ambientais que resultassem não só em um desempenho ambientalmente responsável, como também na otimização de suas relações com as diversas partes interessadas, como cliente, fornecedores e comunidade em geral (Moura, 2000).
Grandes empresas são mais propensas a atender esses requisitos, pois são rigidamente e constantemente fiscalizadas por órgãos responsáveis e, muitas vezes, passam a adotar ferramentas de gestão ambiental baseadas em uma visão estratégica que objetiva cumprir com a legislação ambiental e alcançar, simultaneamente, um melhor desempenho ambiental e econômico, evitando embargos, reduzindo seus custos, e ganhando espaço em um mercado mais exigente ambientalmente (Hahn et al., 2015).

As micro e pequenas empresas (MPEs), no entanto, devido a suas especificidades de gestão, não podem fazer uso dos mesmos mecanismos para minimizar os impactos ambientais de suas atividades. McKeiver \& Gadenne (2005) afirmam que se tornou imperioso dar maior atenção a esse segmento empresarial, atestando a necessidade de estudos que contribuam para o melhor entendimento da relação entre essas especificidades e a adoção de práticas ambientais, procurando aprofundar conhecimentos a respeito dos fatores inerentes ao segmento das MPEs que possam restringir ou estimular ações no campo ambiental.

Ainda que representem $98,5 \%$ do total de empresas no Brasil, respondam por $27 \%$ do Produto Interno Bruto (PIB) e gerem mais da metade dos empregos no país (SEBRAE, 2017), são escassos os estudos que abordam a temática ambiental no 
contexto das MPEs no Brasil. Enquanto que, no contexto internacional, é uma pauta bastante relevante, como mostram os trabalhos de Gunnarsson et al. (2010), Zorpas (2010); Atanase \& Visan (2011), Roxas \& Coetzer (2012), Jorge et al. (2015); Font et al. (2016), Witjes et al. (2017), Graafland (2018), e muitos outros.

Quando se fala em Amazônia, a problemática de escassez de trabalhos é ainda maior. Não tendo sido encontrada nenhuma pesquisa com essa temática durante a realização deste trabalho. Esta foi, então, a maior motivação para a realização deste, que objetiva conhecer como tem se estabelecido a gestão ambiental em micro e pequenas empresas amazônicas, mais especificamente nas empresas localizadas na Região Metropolitana de Belém, que compreende os municípios de Ananindeua, Belém, Benevides, Marituba, Santa Bárbara do Pará, Santa Isabel do Pará e Castanhal.

\section{Revisão bibliográfica}

\subsection{O meio ambiente e as MPES}

Durante as últimas décadas, a discussão acerca da degradação do meio ambiente cresceu até se tornar um tema de maior importância dentre diversos atores da sociedade, como políticos, ONGs e consumidores. Nesse debate, muita responsabilidade é colocada sobre as empresas, que, com os consumidores, muitas vezes são vistas como atores-chave no caminho para o aumento da sustentabilidade ambiental (Haddock-Fraser \& Tourelle, 2010; Sandhu et al., 2010) e desenvolvimento sustentável.

Considerando o estabelecimento e publicidade da Responsabilidade Social Corporativa (RSC), dos relatórios de sustentabilidade e desenvolvimento sustentável (Moore \& Manring, 2009; Wong et al., 2014), parece que o setor corporativo está cada vez mais consciente dos aspectos ambientais e da sustentabilidade de suas operações (Revell et al., 2010; Cassells \& Lewis, 2011; Brammer et al., 2012). Recente relatório da Comissão Europeia (CE) mostra que as grandes empresas são mais propensas a adotar medidas mais eficientes para uso dos recursos, reciclagem, para disponibilizar produtos e serviços ecológicos e implantação de sistema de gestão ambiental do que as MPEs (EC, 2012).

Todavia, embora cada atividade das MPEs represente apenas uma microparcela da poluição global (Schaper, 2002), estima-se que o impacto coletivo delas no meio ambiente seja substancial (Hillary, 2000; Zeng et al., 2011), podendo, inclusive, superar a soma do impacto ambiental das grandes empresas (Hillary, 2000).

$\mathrm{O}$ fato é que as MPEs dominam a arena dos negócios em muitos países (Cassells \& Lewis, 2011). Como consequência, é bastante natural que o grupo das MPEs, como um todo, tenha um impacto considerável no ambiente, embora seja difícil calcular um nível preciso de impacto ambiental para o qual as MPEs dão origem (Revell et al., 2010). Na Europa, foram apresentados números entre $60 \% \mathrm{e}$ $70 \%$ do total dos níveis de poluição (Hillary, 2004; Nordic Council Of Ministers, 2005; OCDE, 2007).

Dada a importância das MPEs para o desenvolvimento sustentável (Revell et al., 2010; Klewitz \& Hansen, 2014), é imprescindível que as operações dessas empresas se tornem ambientalmente responsáveis (Rao et al., 2009) e que se entenda o porquê de algumas MPEs serem mais comprometidas com a sustentabilidade do que outras. 
Por esse motivo, acadêmicos e formuladores de políticas estão reconhecendo, cada vez mais, o papel essencial que as MPEs devem desempenhar para a resolução dos problemas ambientais da atualidade (Aragón-Correa et al., 2008; Blundel et al., 2013).

\subsection{As especificidades das MPEs}

Apesar de sua importância, as MPEs têm sido frequentemente argumentadas como retardatárias quando se trata de compromisso com a sustentabilidade (Revell et al., 2010; EC, 2012; Lewis, 2014). Vários fatores baseados nas características únicas das MPEs foram elencados por Del Brío \& Junquera (2003) e Hillary (2004) para explicar esse fenômeno. Dentre eles, destacam-se as diferenças na estratégia ambiental, incluindo aspectos como recursos financeiros, estrutura organizacional, estilo de gerenciamento e capacidades de produção.

Devido às suas especificidades de gestão, as MPEs não podem fazer uso dos mesmos mecanismos utilizados pelas grandes organizações para minimizar os impactos ambientais causados por suas atividades. Desse contexto, surge a importância de propor investigações que visem ampliar e aprofundar conhecimentos a respeito dos fatores inerentes ao segmento das MPEs e que potencialmente possam restringir ou estimular ações no setor ambiental.

Para Tilley (1999), as dificuldades das MPEs em responder às pressões ambientais estão relacionadas ao fato de que as empresas desse porte não podem simplesmente empregar, em baixa escala, soluções desenvolvidas por, e para, gran- des organizações. Hussey \& Eagan (2007) alegam que tomar ferramentas projetadas para melhorar o desempenho ambiental das grandes organizações e reduzi-las de tamanho para as MPEs pode não ser apropriado. Portanto as MPEs necessitam de suas próprias respostas para a compreensão dos impactos ambientais que elas geram (Tilley, 1999; Hussey \& Eagan, 2007).

As razões para as MPEs se engajarem sustentavelmente são geralmente apresentadas na literatura na forma de três fatores que são frequentemente vistos de forma independente. A competitividade da redução de custos tem sido o fator mais referenciado (Salzmann et al., 2005; Revell \& Blackburn, 2007; Carroll \& Shabana, 2010). Tal fator atesta que empresas que adotam ações ambientalmente responsáveis geram benefícios internos e operacionais diretos relacionados ao uso consciente dos recursos naturais, e aumentam sua vantagem competitiva baseada na redução de custos, diferenciação de produtos, aumento de mercado além outras vantagens econômicas.

Indo além do fator puramente econômico, um segundo fator (Spence et al., 2003; Fuller \& Tian, 2006; Bronn \& Vidaver-Cohen, 2009; Garay \& Font, 2012), que geralmente é usado para entender motivações pró-sustentabilidade, pode ser chamado de legitimação da sociedade, este que atesta que empresas decidem adotar ações de sustentabilidade se cobradas, ou para satisfazer terceiros, como clientes e stakeholders.

Finalmente, e considerando a natureza particular da propriedade e gestão das MPEs, a literatura destaca outro fator que é a expectativa de ganho econômico associado aos valores e estilo de vida de seus proprietários. Esse terceiro fator (Shaw \& Williams, 2004; Carlsen et al., 2008) difere dos 
dois primeiros, pois atesta que o comportamento pró-sustentabilidade advém por motivações pessoais e estilo de vida de seus proprietários e gestores e que, às vezes, pode inclusive ser visto como um conflito na busca exclusiva pelo ganho econômico.

MPEs são, em geral, geridas pelos seus proprietários, que são voltados principalmente para a resolução de problemas do dia a dia. As tomadas de decisões são, às vezes, uma extensão da personalidade e características do proprietário-gerente, frequentemente moldando à cultura das MPEs e decretando valores e hábitos que muitas vezes não visam unicamente ao lucro (Fassin et al., 2011).

\subsection{Dificuldades de implantação da gestão ambiental nas MPES}

Muitas são as barreiras que dificultam ou impedem a adoção de atividades de gestão ambiental ou práticas ecologicamente corretas por parte das MPEs. Martins et al. (2016) apresenta uma sistematização das principais barreiras identificadas na literatura, elas são: a falta de conscientização dos benefícios da gestão ambiental; a falta de conhecimento dos dirigentes no campo ambiental; inconsciência de danos ambientais causados por suas atividades; a falta de recursos financeiros para adoção das práticas; a carência de recursos humanos conhecedores de gestão ambiental; a baixa pressão dos clientes; e a falta de informações e tempo dos dirigentes.

Fernández-Viñe et al., (2010) argumentam que os dirigentes das MPEs não acreditam que um melhor desempenho ambiental possa contribuir com o aumento de vendas, com melhoria na competitividade ou na motivação de funcionários, pois, para eles, o fundamental é a sobrevivência do negócio
(Trianni et al., 2013), já que, diferente das grandes empresas, estas não estão totalmente consolidadas no mercado.

A carência de recursos financeiros por parte das MPEs é outro fator que está intimamente ligada ao escasso desenvolvimento de práticas ambientais (Redmond et al., 2008; Gadenne et al., 2009; Brammer et al., 2012; Tamajón \& Aulet, 2013; Tikul, 2014). Os dirigentes dessas empresas acreditam que não possuem os recursos para fazer a mudança necessária (Johannson, 1997), especialmente pelo fato de que o custo de implantação de tecnologias de produção mais limpa é consideravelmente mais alto quando comparado ao de tecnologias convencionais (Shi et al., 2008).

Dessa forma, a restrição financeira reduz a probabilidade das MPEs gerarem inovações ambientais (Cuerva et al., 2014). Trianni et al. (2013) acrescentam ainda que alguns dirigentes de MPEs detém-se mais por medo da existência de custos ocultos do que pelos custos do investimento inicial na adoção de medidas relacionadas à implantação da gestão ambiental.

Mais do que os recursos financeiros, os recursos humanos $(\mathrm{RH})$ podem ser um fator preponderante para a implementação de sistemas de gestão ambiental nas MPEs (Hillary, 2004). A carência de RH (Hillary, 2004; Studer et al., 2006; Redmond et al., 2008) e a natureza multifuncional do staff tornam-se de importância crescente com a diminuição do tamanho da empresa, não somente para a implementação, mas também para a manutenção dos sistemas de gestão ambiental (Hillary, 2004).

Tikul (2014) faz referência também ao fato de muitas MPEs assumirem a posição de que não possuem funcionários suficientes para a formação de equipes com vistas ao treinamento ambiental. 
Pois, nessas empresas, normalmente o funcionário já exerce mais de uma função e o seu mau desempenho pode afetar a organização como um todo.

Os dirigentes das MPEs têm pouco ou nenhum conhecimento no campo da gestão ambiental (Shi et al., 2008; Brammer et al., 2012). Questões ambientais de nível global, como mudanças climáticas, são frequentemente tratadas por dirigentes das MPEs como um tema abstrato, distante no espaço e no tempo, e, para com as quais, suas empresas têm pouca responsabilidade (North \& Nurse, 2014).

Muitas vezes, essas atitudes são similares quando relacionadas ao meio ambiente, quando as MPEs consideram medidas de gestão ambiental inapropriadas ou desnecessárias (Tikul, 2014), desconhecendo a extensão de seus impactos (Zorpas, 2010) ou adotando a visão de que seus negócios não causam qualquer dano ambiental. E por julgarem seus impactos como ínfimos ou inexistentes (Perez-Sanchez et al., 2003; Redmond et al., 2008; Battisti \& Perry, 2011), a maioria das empresas de menor porte não destina recursos específicos para a resolução de problemas ambientais e implantação de tecnologias limpas (Fernández-Viñe et al., 2010).

A falta de informações adequadas e acessíveis sobre como melhorar as atividades ambientais da empresa é uma variável importante a se considerar (Schaper, 2002; Mckeiver \& Gadenne, 2005). A informação contribui para o aumento da compreensão sobre problemas ambientais, podendo agir como um estímulo à melhoria de desempenho, primeiro ao demonstrar porque ações nesse sentido são necessárias e, posteriormente, prover meios pelos quais as empresas possam aprender como e quando agir (Schaper, 2002).

Outra barreira constatada por Hillary (2004) é a falta de exigência do mercado. Quando os clientes demonstram falta de interesse ou estão satisfeitos com o desempenho ambiental das MPEs, emerge uma grande barreira à adoção de práticas ambientais, podendo ser considerada, em alguns casos, como a mais importante pelas empresas.

Essa afirmação corrobora com a visão de Tamajón \& Aulet (2013) que classificam como obstáculo da gestão ambiental a falta de indagação, por parte dos clientes, sobre medidas de responsabilidade ambiental adotadas pelas MPEs e com a de Battisti \& Perry (2011) que mencionam que os dirigentes de MPEs consideram a implantação de práticas ambientais apenas como custo, tendo em vista a menor exposição às pressões de mercado, em relação às grandes empresas. Portanto a conscientização ecológica dos clientes torna-se de grande importância para o aumento da adoção de tecnologias limpas e práticas sustentáveis (Studer et al., 2006; Cuerva et al., 2014).

Heras \& Arana (2010) chamam a atenção ainda, para a barreira legal, afirmando que as MPEs têm dificuldade no entendimento e cumprimento da legislação ambiental. Além do mais, muitas facilidades como financiamento tecnológico, oportunidades de mercado, incentivos relacionados e transferência de tecnologias ambientalmente corretas também não são de conhecimento dos dirigentes (Rao et al., 2006).

\subsection{Gestão ambiental empresarial no contexto amazônico}

Considerada como nova função empresarial, a gestão ambiental possibilita que as empresas administrem adequadamente suas relações com o meio ambiente ao avaliarem e corrigirem danos ambientais do presente ou evitarem problemas 
futuros, além de integrarem todos os setores da empresa aos imperativos ambientais (Sanches, 2000). Conforme conceituação de Barbieri (2016), a gestão ambiental corresponde ao conjunto de diretrizes e atividades administrativas e operacionais realizadas pela empresa para abordar problemas ambientais decorrentes da sua atuação ou para evitar que eles ocorram no futuro.

A Região Amazônica apresenta baixo percentual de implantação de gestão ambiental e de emissão de certificados ambientais (Mendonça \& Nahum, 2018), ainda que em estados como Pará e, principalmente, Amazonas, com a Zona Franca de Manaus, apresentem grandes empresas, multinacionais e exportadoras.

Supõe-se que a Região ainda sofra com modelos econômicos implantados na Amazônia nas décadas de 1960 e 1970 e com achismo de que o crescimento econômico é contrário à preservação e conservação dos recursos naturais. Observa-se por meio de breve pesquisa que a complexidade ambiental se encontra bastante ausente nas Políticas de Gestão Ambiental dos Estados e que há ausência de percepção dos danos ambientais como problema de ordem local e global (Silva, 2002), tanto do governo quanto das empresas. E não só isso, de modo geral, não há conscientização populacional das questões ambientais. As empresas não sofrem cobrança por práticas ecologicamente corretas, nem são privilegiadas quando à adotam.

Os estados de Amapá, Roraima e Rondônia são os únicos do Brasil a não possuírem nenhuma empresa certificada ambientalmente. $\mathrm{O}$ estado do Pará, do qual a Região Metropolitana de Belém faz parte, mesmo sendo referência no setor da mineração e da indústria extrativa, possui apenas 12 certificados emitidos, todos eles para empresas de grande porte e multinacionais que tem como premissa atender requisitos de países estrangeiros objetivando exportar sua produção (Mendonça \& Nahum, 2018).

O estado do Amazonas, ainda que detentor da Zona Franca de Manaus, zona industrial brasileira localizada em Manaus, criada para impulsionar o desenvolvimento econômico da Amazônia Ocidental, possui apenas 86 certificados emitidos, número muito baixo, se consideradas as 700 indústrias abrigadas no Polo (Serafico \& Serafico, 2005).

Com total de 101 certificados ativos, considerando todos os estados da Região Amazônica, nenhuma empresa realizou a transição para a ISO 4001:2015 (ABNT, 2015a). Dado preocupante, se considerado que existe menos de um ano para o término do prazo de adequação, e que este é um processo demorado até a certificação.

Quando se fala de MPEs, o panorama de implantação da gestão ambiental e de práticas ambientalmente corretas na Amazônia é ainda mais obscuro, não tendo sido encontrado na literatura trabalhos que versem sobre essa pauta.

De acordo com Miles et al. (1999), para a adoção das normas da série ISO 14000, é necessário o desembolso de significativos recursos financeiros, e a maioria das MPEs, além de não terem esses recursos internamente, necessitam de "onerosas" ajudas externas, uma vez que seus funcionários não possuem as capacidades e habilidades técnicas para a implantação e manutenção de sistemas de gestão ambiental.

Johannson (1997) menciona também que a quantidade de recursos financeiros exigida para a certificação das normas ISO 14000 é ainda mais significativa se compararmos com o nível de fa- 
turamento das MPEs, podendo inclusive superar a margem de lucro dessas empresas.

Então, a partir desta pesquisa, considerando o inexistente número de certificações em MPEs, as dificuldades de adoção da gestão ambiental e a falta de discussão desta pauta na literatura e na sociedade amazônica como um todo, optou-se por abordar a adoção de práticas ambientais da forma mais simples e básica para, assim, obter-se o panorama atual da gestão ambiental nas MPEs amazônicas.

\section{Metodologia}

\subsection{Região de estudo}

A Grande Belém localiza-se na região mais dinâmica do estado do Pará e, juntamente com o município de Barcarena, integra o segundo maior parque industrial da Amazônia (Ferreira, 2003). A economia belenense baseia-se primordialmente nas atividades do comércio, serviços e turismo, embora seja também desenvolvida a atividade industrial com grande número de indústrias alimentícias, navais, metalúrgicas, pesqueiras, químicas e madeireiras.

Historicamente, a Região Metropolitana de Belém, também conhecida como "Metrópole da Amazônia" e que compreende os municípios de Ananindeua, Belém, Benevides, Marituba, Santa Bárbara do Pará, Santa Isabel do Pará e Castanhal, constitui-se na principal via de entrada na Região Norte do Brasil, com uma posição geográfica privilegiada. Devido ao intenso processo de conurbação, hoje é um dos maiores aglomerados urbanos da Região Norte.

No estado do Pará, mais de $90 \%$ dos empreendimentos são qualificados como micro ou pequenas empresas e responderam, em 2015 , por $40 \%$ dos empregos na indústria, $68 \%$ no comércio e $43 \%$ no setor serviços, perfazendo um total de 57.043 MPEs registradas e ativas nos setores de indústria, comércio e serviço (Silva et al., 2016). Segundo o IPEA (2010), Belém agrega os maiores índices de infraestrutura urbana, de serviços e de concentração econômica.

\subsection{Método}

Para obter o panorama da gestão ambiental em micro e pequenas empresas amazônicas, visando conhecer as motivações e entraves na adoção de práticas ecologicamente corretas, bem como conhecer as práticas que tenham o compromisso com a sustentabilidade e que já vem sendo adotadas na Região, realizou-se uma abordagem quantitativa. Isto se deveu principalmente à natureza exploratória da pesquisa, mas também devido ao fato de que muitas pesquisas na área usam estudos de caso ou amostras limitadas para estudar essas questões (Hofmann et al., 2012). E, neste estudo, buscou-se tirar conclusões sobre as MPEs da maneira mais geral possível.

\subsubsection{Amostragem}

Uma pesquisa por questionário, denominada survey, foi realizada durante o período de dezembro de 2017 a abril de 2018. Por uma questão de acessibilidade, e-mails de empresas da Região Metropolitana de Belém foram obtidos a partir de banco de dados existente, assim como preconizado por Jansson et al. (2017).

Inicialmente, foi realizado um questionário piloto para verificar se os resultados a serem obti- 
dos responderiam aos objetivos de pesquisa, se os questionados entenderiam as perguntas e saberiam como responder, e se a pesquisa teria resposta e aceitação por parte das empresas.

A partir do feedback do questionário piloto, optou-se pela redução do número de questões do questionário, simplificação de linguagem técnica e pela abordagem de práticas mais simples e básicas de gestão ambiental, tendo em vista que a inserção destas nas MPEs amazônicas ainda se encontra em fase inicial ou de não aplicação.

No total, o questionário via e-mail foi enviado para 580 empresas de diferentes setores. Destes, 93 e-mails retornaram por serem endereços inativos, e 102 questionários preenchidos foram devolvidos, resultando em uma taxa de resposta total de $17 \%$. Considerando o curto tempo de resposta e a amplitude das empresas que recebem o link da pesquisa, a taxa de resposta foi considerada aceitável.

Das respostas retornadas, $54 \%$ eram de microempresas ( 0 a 10 funcionários) e $46 \%$ de pequenas empresas (10 a 49 funcionários), definição de porte adotada por SEBRAE (2013) (Tabela 1). O percentual próximo, entre resposta de micro e pequenas empresas, configura-se fato não esperado, tendo em vista que o número de microempresas ativas é bastante superior às pequenas.

TABELA 1 - Enquadramento das empresas por número de funcionários.

\begin{tabular}{lcc}
\multicolumn{1}{c}{ Enquadramento } & F & $\mathbf{f ( \% )}$ \\
\hline Micro (1-9 funcionários) & 55 & $\mathbf{5 3 , 9 2}$ \\
Pequena (10-49 funcionários) & 47 & $\mathbf{4 6 , 0 8}$ \\
\hline Total & 102 & $\mathbf{1 0 0}$ \\
\hline
\end{tabular}

Em comparação com o quantitativo de empresas ativas registradas na Região Metropolitana de
Belém, a amostra foi estimada como representativa das MPEs na região de estudo.

Buscou-se amostrar empresas dos mais diferentes ramos quanto possíveis para, assim, obter-se a generalização do estudo dentro da região estudada (Tabela 2). Dos 102 questionários válidos, o ramo Restaurante foi o melhor representado, seguido por "Outros" que perfazem empresas de variados ramos, como: Lojas de varejo, Clínicas, Escolas etc. Os questionários foram enviados de forma aleatória, especificando-se apenas o porte. E foram tabulados a partir da obtenção de questionários válidos.

Toda e qualquer empresa produz algum tipo de impacto em sua atividade e, ainda que de forma TABELA 2 - Ramo das empresas respondentes.

\begin{tabular}{lcc}
\hline \multicolumn{1}{c}{ Ramo } & F & $\mathbf{f ( \% )}$ \\
\hline Restaurante & 29 & $\mathbf{2 8 , 4 3}$ \\
Supermercado & 14 & $\mathbf{1 3 , 7 3}$ \\
Padaria & 3 & $\mathbf{2 , 9 4}$ \\
Indústria & 4 & $\mathbf{3 , 9 2}$ \\
Lava a Jato & 3 & $\mathbf{2 , 9 4}$ \\
Transportadora & 6 & $\mathbf{5 , 8 8}$ \\
Posto de Gasolina & 9 & $\mathbf{8 , 8 2}$ \\
Loja de Produto Agrícola & 4 & $\mathbf{3 , 9 2}$ \\
Criação de animais & 8 & $\mathbf{7 , 8 4}$ \\
Recapagem/ Borracharia & 1 & $\mathbf{0 , 9 8}$ \\
Outros & 21 & $\mathbf{2 0 , 5 9}$ \\
\hline Total & 102 & $\mathbf{1 0 0 , 0 0}$ \\
\hline
\end{tabular}

minimizada, utiliza recursos naturais para existir. Esse fato e o interesse pela generalização do resultado justificam as perguntas generalistas feitas nos questionários, passíveis de serem respondidas por todos os ramos empresariais. 


\subsubsection{Medidas}

Todas as medidas foram avaliadas usando uma escala de Likert (Likert, 1932) variando de 1 , nada importante, a 7 , extremamente importante; ou 1, discordo completamente, a 5, concordo completamente. E versaram sobre: a importância da preservação ambiental para o desenvolvimento estratégico da empresa; práticas ecologicamente corretas adotadas pela empresa; fatores que fazem ou fariam a empresa adotar práticas ecologicamente corretas; e entraves para adoção de práticas ecologicamente corretas pela empresa.

Ressalta-se, desde já, que as conclusões recolhidas nos questionários resultam das respostas dos inquiridos, por isso, dependem da fiabilidade destas. Na medida do possível, tentou-se combinar os resultados do inquérito por questionário com a observação participante e as referências bibliográficas e documentais consultadas.

\section{Resultados e discussão}

A análise e discussão dos dados apresentados neste capítulo incluem a caracterização das empresas respondentes, as percepções de seus gestores acerca da importância da preservação ambiental para o desenvolvimento estratégico das empresas, as práticas ecologicamente corretas adotadas por essas empresas, os fatores que fazem ou fariam adotar práticas ecologicamente corretas, as barreiras encontradas por essas empresas para adoção de práticas ecologicamente corretas e, finalmente, o panorama da gestão ambiental em micro e pequenas empresas amazônicas.

\subsection{Importância da preservação ambiental para o desenvolvimento estratégico da empresa}

A partir dos resultados deste estudo, pode-se atestar que, ainda que a pauta "Preservação Ambiental" seja bastante atual e esteja sendo difundida nos mais diferentes meios e setores, esta não tem sua importância embutida no planejamento do desenvolvimento estratégico das empresas amazônicas, como se observa na Tabela 3, em que "1" representa "Nada importante" e "7", "Extremamente importante".

TABELA 3 - Grau de importância da preservação ambiental para o desenvolvimento estratégico das empresas respondentes, segundo escala de Likert.

\begin{tabular}{ccc}
\hline Grau & F & $\mathbf{f}(\mathbf{\%})$ \\
\hline $\mathbf{1}$ & 61 & 59,80 \\
$\mathbf{2}$ & 28 & 27,45 \\
$\mathbf{3}$ & 7 & 6,86 \\
$\mathbf{4}$ & 4 & 3,92 \\
$\mathbf{5}$ & 0 & 0,00 \\
$\mathbf{6}$ & 1 & 0,98 \\
$\mathbf{7}$ & 1 & 0,98 \\
\hline Total & $\mathbf{1 0 2}$ & $\mathbf{1 0 0 , 0 0}$ \\
\hline
\end{tabular}

O planejamento estratégico empresarial é o processo no qual se decide como colocar em prática passos e abordagens administrativas que visam o crescimento dos negócios de forma competitiva (Anthony \& Govindarajan, 2006; Thompson et al., 2008). Está diretamente relacionado à gestão estratégica e define a visão de futuro, a missão da instituição, quais objetivos e metas devem ser alcançados, que estratégias devem ser utilizadas, quais atividades 
devem ser executadas e quais recursos são necessários (financeiros, materiais e humanos) para que os propósitos da organização sejam realmente satisfeitos (Falsarella \& Jannuzzi, 2017).

Numa escala Likert variando de 1 a 7 em grau de importância crescente, a obtenção de média 1,64, moda 1, e variância de 1,094 representam que quase nenhuma importância é dada à preservação ambiental na definição de objetivos e diretrizes adotados pelas empresas respondentes. O meio ambiente ainda não está presente, seja na filosofia, seja nos métodos produtivos adotados na maioria das empresas.

Esse fato pode justificar-se, primeiramente, porque MPEs ignoram o planejamento estratégico formalizado ou fazem o mínimo uso dele (Huang \& Brown, 1999; Leone, 1999; Gray \& Mabey, 2005; Torrès \& Julien, 2005). Empresas desse porte não necessitam desenvolver técnicas complexas de gestão, mas, sim, formular um planejamento adequado a suas especificidades (Terence \& Escrivão, 2001), no qual, a partir do resultado desta pesquisa, pode-se concluir que a preservação do meio ambiente quase não está inserida.

Ademais, MPEs, diferente de grandes empresas, estão focadas no crescimento econômico, estabelecimento no mercado e na fidelização de consumidores (Gray \& Mabey, 2005). Não sendo o fator ambiental julgado prioritário para se alcançar tais objetivos, considerando a pequena ou nula exigência do mercado amazônico por posturas ecologicamente responsáveis, como veremos a seguir.

\subsection{Práticas ecologicamente corretas adotadas pela empresa.}

Como apresentado na Tabela 4, em que os dados foram obtidos por escala Likert variando de
1 a 7, em que 1 representa "Discordo completamente" e 7, "Concordo completamente", as práticas majoritariamente adotas pelas MPEs respondentes estão diretamente ligadas a fatores econômicos e redução de custos, assim como resultados obtidos por Font et al. (2016).

A busca pela redução de custos por diminuição do uso de recursos naturais faz com que "economia de energia elétrica" e "economia do consumo de água" sejam algumas das principais práticas ecologicamente adotadas pelas empresas respondentes, aliadas ao "cumprimento de legislações ambientais".

O cumprimento das leis é visto como meio de não gerar multas e evitar embargos à atividade produtiva. E, nesse ponto, é importante ressaltar que a legislação ambiental na região estudada é uma das mais completas do país e a Secretaria de Meio Ambiente uma das mais atuantes, tanto no que se diz respeito ao licenciamento ambiental quanto à fiscalização ambiental em áreas urbanas e rurais.

As média e dispersão dos fatores analisados encontram-se na Tabela 5. A partir dessa análise estatística, percebe-se que as médias representam bem a percepção das empresas em relação às práticas ecologicamente corretas adotadas. E que, ainda que de diferentes portes e ramos, as empresas respondentes possuem, maioritariamente, o mesmo perfil ambiental. Excetuando algumas poucas em que foi possível observar claramente a influência da motivação pessoal do gestor em relação ao meio ambiente na adoção de práticas e objetivos ecologicamente corretos. 
TABELA 4 - Práticas ecologicamente corretas adotadas pelas empresas respondentes, segundo escala de Likert.

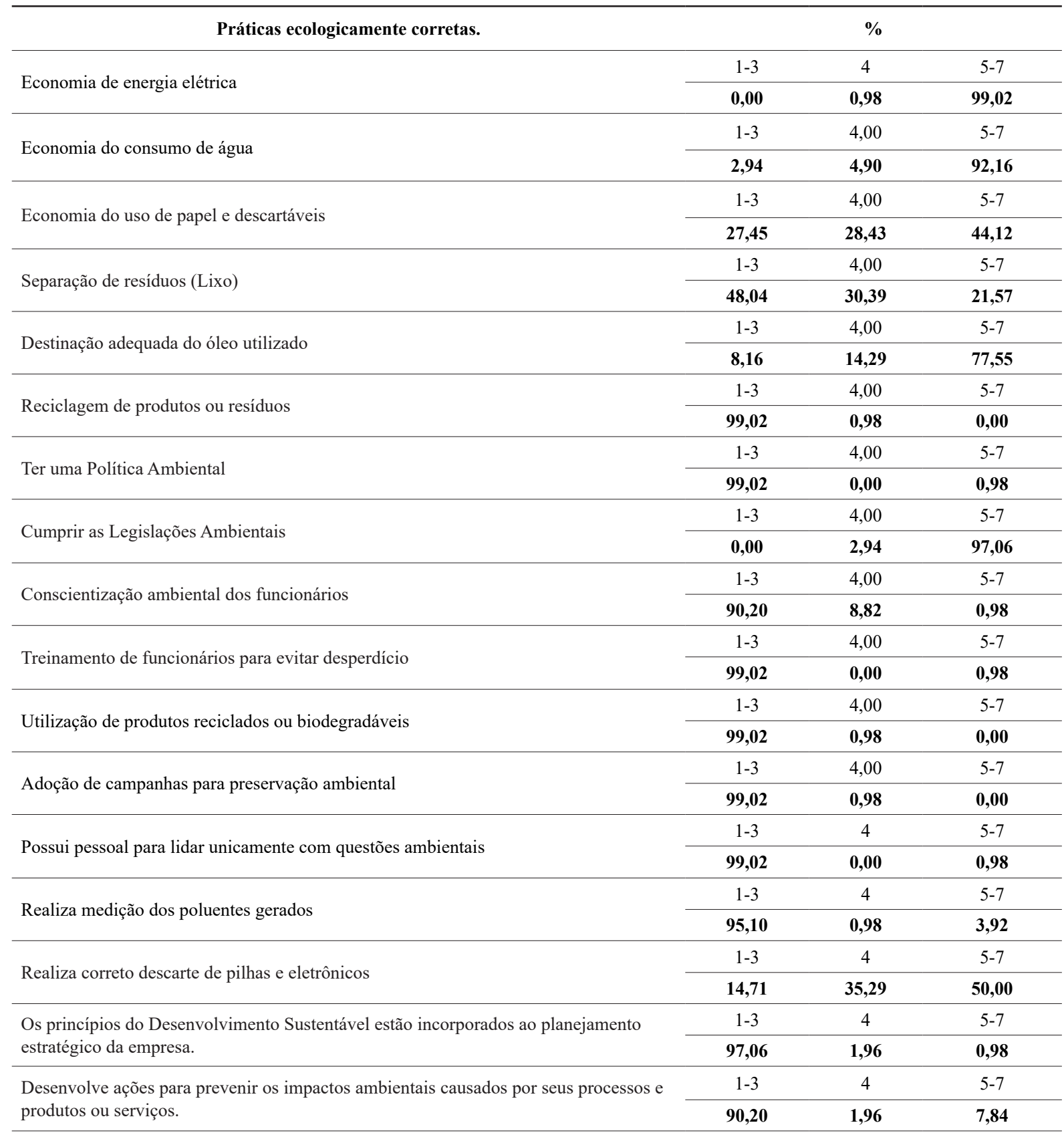




\begin{tabular}{|c|c|c|c|}
\hline \multirow{2}{*}{ Produz estudos de impacto em toda a cadeia produtiva } & $1-3$ & 4 & $5-7$ \\
\hline & 96,08 & $\mathbf{0 , 0 0}$ & 3,92 \\
\hline Investe em tecnologia para redução da geração de resíduos & $1-3$ & 4 & $5-7$ \\
\hline
\end{tabular}

TABELA 5 - Média e Dispersão das Práticas ecologicamente corretas adotadas pelas empresas respondentes.

\begin{tabular}{|c|c|c|c|c|c|c|}
\hline Práticas ecologicamente corretas & $\mathbf{n}$ & Média & Moda & Med & Des. $\mathbf{P}$ & Var \\
\hline Economia de energia elétrica & 102 & 6,81 & 7 & 7 & 0,521 & 0,269 \\
\hline Economia do consumo de água & 102 & 6,51 & 7 & 7 & 1,041 & 1,073 \\
\hline Economia do uso de papel e descartáveis & 102 & 4,74 & 7 & 4 & 2,073 & 4,253 \\
\hline Separação de resíduos (Lixo) & 102 & 3,17 & 1 & 4 & 2,308 & 5,276 \\
\hline Destinação adequada do óleo utilizado & 49 & 5,86 & 7 & 7 & 1,803 & 3,184 \\
\hline Reciclagem de produtos ou resíduos & 102 & 1,08 & 1 & 1 & 0,364 & 0,131 \\
\hline Ter uma Política Ambiental & 102 & 1,06 & 1 & 1 & 0,594 & 0,349 \\
\hline Cumprir as Legislações Ambientais & 102 & 6,89 & 7 & 7 & 0,525 & 0,273 \\
\hline Conscientização ambiental dos funcionários & 102 & 1,40 & 1 & 1 & 1,046 & 1,084 \\
\hline Treinamento de funcionários para evitar desperdício & 102 & 1,06 & 1 & 1 & 0,594 & 0,349 \\
\hline Utilização de produtos reciclados ou biodegradáveis & 102 & 1,14 & 1 & 1 & 0,488 & 0,236 \\
\hline Adoção de campanhas para preservação ambiental & 102 & 1,07 & 1 & 1 & 0,379 & 0,142 \\
\hline Possui pessoal para lidar unicamente com questões ambientais & 102 & 1,06 & 1 & 1 & 0,594 & 0,349 \\
\hline Realiza medição dos poluentes gerados & 102 & 1,26 & 1 & 1 & 1,202 & 1,430 \\
\hline Realiza correto descarte de pilhas e eletrônicos & 102 & 4,73 & 4 & 4,5 & 1,862 & 3,434 \\
\hline $\begin{array}{l}\text { Os princípios do Desenvolvimento Sustentável estão incorporados ao } \\
\text { planejamento estratégico da empresa }\end{array}$ & 102 & 1,12 & 1 & 1 & 0,722 & 0,516 \\
\hline $\begin{array}{l}\text { Desenvolve ações para prevenir os impactos ambientais causados por } \\
\text { seus processos e produtos ou serviços }\end{array}$ & 102 & 1,60 & 1 & 1 & 1,672 & 2,770 \\
\hline Produz estudos de impacto em toda a cadeia produtiva & 102 & 1,24 & 1 & 1 & 1,170 & 1,356 \\
\hline Investe em tecnologia para redução da geração de resíduos & 102 & 1,31 & 1 & 1 & 1,251 & 1,549 \\
\hline
\end{tabular}


A maior variância em fatores como "economia do uso de papel e descartáveis", "separação de lixo", "destinação adequada do óleo utilizado", e "correto descarte de pilhas e eletrônicos" está diretamente ligado a incentivos governamentais ou privados realizados apenas em alguns bairros dentro da região de estudo.

A coleta seletiva, que incentiva a separação do lixo, não ocorre em todos os bairros da região e muito dessa coleta advém de cooperativas de catadores como a CONCAVES que coletam resíduos nas próprias empresas e que recebem incentivos governamentais.

No que diz respeito a destinação adequada de óleo, é importante ressaltar que apenas responderam as empresas que fazem uso de tal insumo. A alta variância desse fator deve-se provavelmente a empresas como a "Cata Óleo", privada, sem apoio governamental e com fins lucrativos, que faz recolhimentos nas próprias empresas de todo óleo usado no processo produtivo. Todavia, a "Cata Óleo" possui uma zona de ação que não inclui toda a região pesquisada.

O correto descarte de pilhas e eletrônicos é incentivado por locais específicos de coletas encontrados em supermercados, universidades e centros de pesquisas, entretanto estes também não são encontrados em todos os bairros da região estudada.

Quando se aborda a "economia do uso de papel e descartáveis", a variância deve-se à adoção por certas empresas de ações como "use seu próprio copo" ou “é mesmo necessário imprimir?", enquanto que outras empresas ou desconhecem tais ações, ou minimizam sua importância.

A pouca adoção de práticas ecologicamente corretas pelas MPEs preocupa, pois estas correspondem a $90 \%$ dos empreendimentos ativos no estado do Pará, onde localiza-se a região estudada. $E$ ainda que individualmente essas empresas não representem grandes impactos ambientais, juntas, elas são responsáveis por um impacto considerável.

\subsection{Fatores que fazem ou fariam as empresas adotarem práticas ecologicamente corretas}

Dentre os fatores que fazem ou fariam as empresas respondentes adotarem práticas ecologicamente corretas (Tabela 6) estão, principalmente: a "exigência do mercado consumidor", a "adequação à legislação ambiental", o "não pagamento de multas ambientais", a "redução de impostos por boas práticas ambientais" e a "redução de custos". Assim como as práticas ecologicamente corretas já adotadas nos mostram, os fatores que fazem ou fariam as empresas as adotarem também estão diretamente relacionados a questões econômicas. E que nos leva a concluir que os consumidores e o governo são os principais agentes de uma possível mudança desse cenário.

A média e dispersão dos dados apresentados na Tabela 7 confirmam os valores absolutos obtidos na Tabela 6 e que, para a maior parte dos fatores observados, a média encontrada é representativa do todo.

Os fatores "exigência de fornecedores", "marketing ambiental" e "adoção por parte dos concorrentes" foram os que apresentaram maior variância em relação à média. Esse fato deve-se a que algumas das empresas não movidas por motivações pessoais de seus gestores, e/ou estão em nível superior de estabelecimento no mercado em relação às outras, ou ainda possuem consumidores mais exigentes, que justifiquem a necessidade da adoção de marketing ambiental e uma rede ecologicamente 
correta em conjunto com os fornecedores. Ainda assim, de forma geral, todas as MPEs respondentes são movidas e motivadas pela redução de custos e atendimento aos clientes.

Ao contrário do que se esperava, o fator "adoção por parte dos concorrentes" se mostrou pouco motivacional. Na literatura disponível sobre o desempenho sustentável das MPEs, não há concordância sobre a relação entre o desempenho ambiental e desempenho competitivo nas MPEs. Estudos, como Link \& Naveh (2006), Sarkis \& Dijkshoorn (2007) ou Iraldo et al. (2009), estabeleceram que a implantação da gestão ambiental não estava associada a melhores desempenhos nos negócios, enquanto que o estudo de Jorge et al. (2015) indica que as MPEs que desenvolvem práticas ambientais experimentam uma vantagem na busca por competitividade.

A adoção de práticas ecologicamente corretas pode representar para as MPEs, oportunidades para alcançar vantagens competitivas no mercado e, portanto, melhorar o seu desempenho em longo prazo, resultado do compromisso com o meio ambiente e a gestão de práticas voltadas para o respeito, proteção e promoção do ambiente (Aragon-Correa et al., 2008; Husillos \& Alvarez-Gil, 2008).

Tabela 6 - Fatores que fazem ou fariam as empresas respondentes adotarem práticas ecologicamente corretas, segundo escala de Likert.

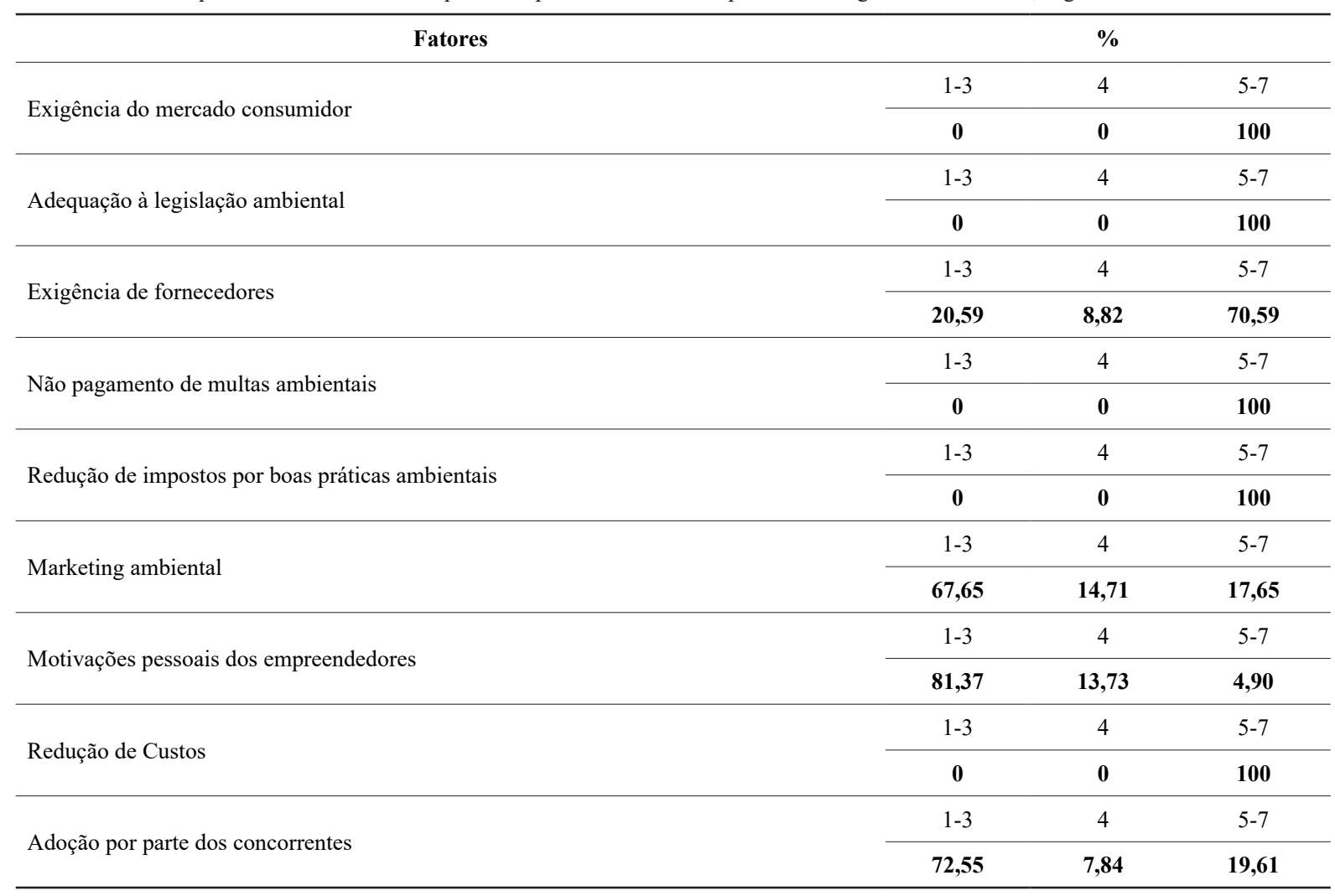


TABELA 7 - Média e Dispersão dos Fatores que fariam ou fazem as empresas respondentes adotarem práticas ecologicamente corretas.

\begin{tabular}{|c|c|c|c|c|c|c|}
\hline Fatores & $\mathbf{n}$ & Média & Moda & Med & Des. P & Var \\
\hline Adequação à legislação ambiental & 102 & 6,89 & 7 & 7 & 0,342 & 0,116 \\
\hline Exigência de fornecedores & 102 & 4,82 & 6 & 5 & 2,041 & 4,126 \\
\hline Não pagamento de multas ambientais & 102 & 6,97 & 7 & 7 & 0,221 & 0,048 \\
\hline Marketing ambiental & 102 & 2,39 & 1 & 1 & 2,116 & 4,434 \\
\hline Motivações pessoais dos empreendedores & 102 & 1,75 & 1 & 1 & 1,367 & 1,852 \\
\hline Redução de Custos & 102 & 6,97 & 7 & 7 & 0,170 & 0,029 \\
\hline
\end{tabular}

\subsection{Barreiras para adoção de práticas ecologicamente corretas pelas empresas}

As principais barreiras encontradas pelas MPEs respondentes para a adoção de práticas ecologicamente corretas foram: "alto custo de implantação", "falta de incentivo financeiro ou governamental", "falta de interesse ou cobrança por parte dos consumidores" e "falta de conhecimentos das práticas que devem ser adotadas". E, assim como nas questões anteriores, as barreiras encontradas também estão relacionadas à questão econômica, como se pode observar na Tabela 8 .

Schleich \& Gruber (2008) e Schleich (2009) concluíram em seus estudos sobre adoção de práticas energéticas que as barreiras econômicas muitas vezes estão associadas à barreira da informação. Eles afirmam que a falta de informação dos gestores das MPEs sobre as boas práticas, e o custo-benefício da adoção destas, é o principal fator que explica as baixas taxas de adoção nessas empresas.
As médias e dispersão das barreiras para a implantação de práticas ecologicamente corretas adotadas pelas empresas estão apresentadas na Tabela 9.

Dentre todos os fatores abordados no questionário, as barreiras foram as que apresentaram menor variância em relação à média obtida. Fato que nos permite concluir que a maioria das empresas concordam entre si sobre quais são as barreiras enfrentadas para a adoção de práticas ecologicamente corretas.

É importante ressaltar que as razões para que as MPEs não adotem boas práticas ambientais, maximizarem barreiras e não realizarem melhorias ambientais em seus processos produtivos podem estar relacionadas à falta de consciência das vantagens advindas da implementação da gestão ambiental em suas atividades de negócios (Hillary, 2004; Massoud et al., 2010; Brammer et al., 2012) e/ ou à percepção de que essas atividades são onerosas (Tilley, 1999; Simpson et al., 2004; Shi et al., 2008; Tamajón \& Aulet, 2013). 
TABELA 8 - Barreiras para a implantação de práticas ecologicamente corretas adotadas pelas empresas respondentes, segundo escala de Likert.

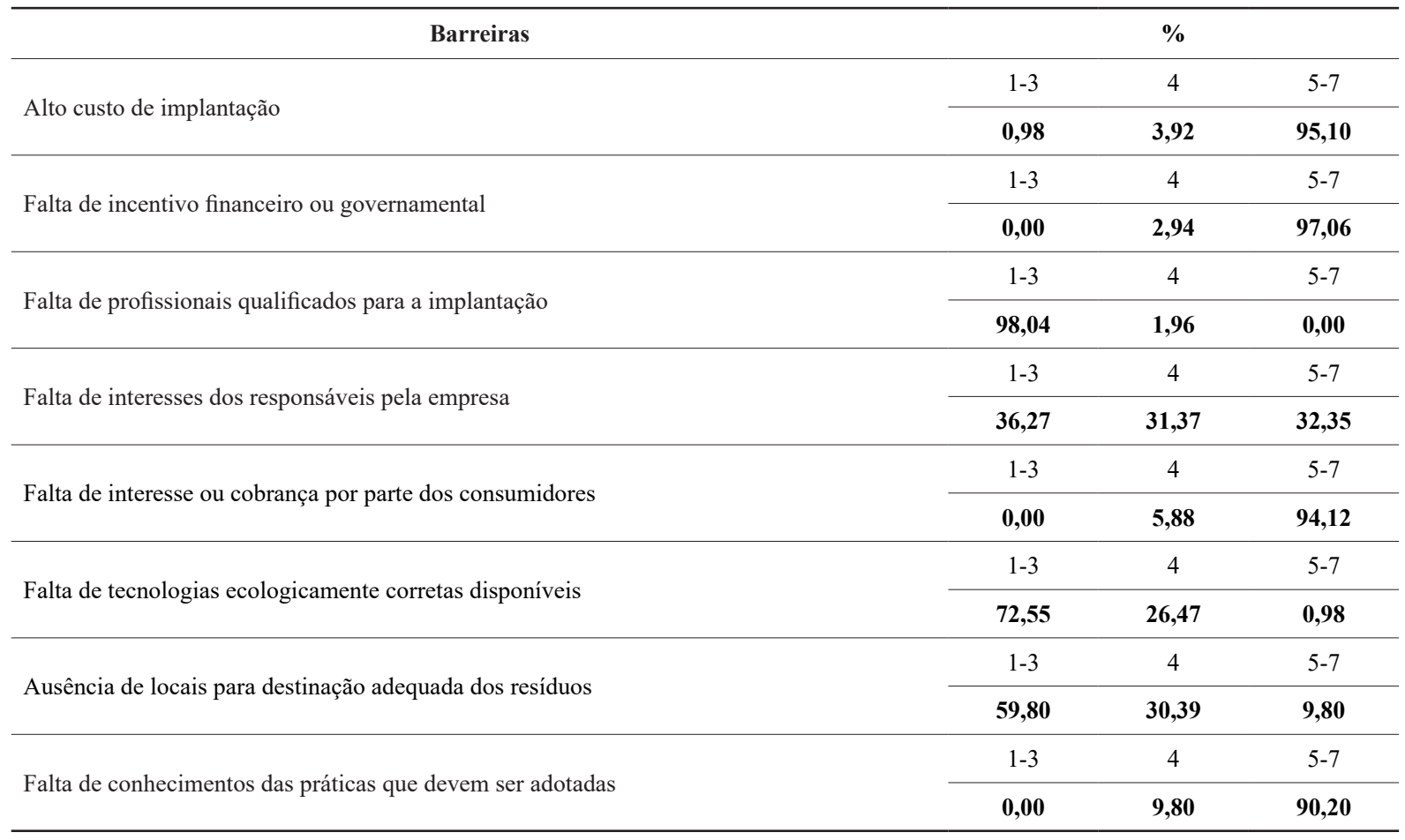

TABELA 9 - Média e dispersão das barreiras para a implantação de práticas ecologicamente corretas adotadas pelas empresas respondentes.

\begin{tabular}{|c|c|c|c|c|c|c|}
\hline Barreiras & $\mathbf{n}$ & Média & Moda & Med & Des. P & Var \\
\hline Falta de profissionais qualificados para a implantação & 102 & 1,64 & 1 & 1 & 0,781 & 0,604 \\
\hline Falta de interesses dos responsáveis pela empresa & 102 & 4,17 & 4 & 4 & 1,935 & 3,708 \\
\hline Falta de tecnologias ecologicamente corretas disponíveis & 102 & 2,22 & 1 & 2 & 1,340 & 1,777 \\
\hline Ausência de locais para destinação adequada dos resíduos & 102 & 3,01 & 4 & 3 & 1,346 & 1,794 \\
\hline Falta de conhecimentos das práticas que a serem adotadas & 102 & 6,12 & 7 & 6 & 0,957 & 0,908 \\
\hline
\end{tabular}


A responsabilidade ambiental é considerada uma carga extra à empresa (Rutherfoord et al., 2000; Studer et al., 2006) que, em muitos casos, não pode ser repassada aos clientes (Tilley, 1999; Simpson et al., 2004), levando os gestores a crer que, em uma relação custo versus benefício, a vantagem da melhoria ambiental tende a ser superada pelo total de gastos.

As barreiras identificadas por esta pesquisa podem servir como subsídio para a formulação de políticas públicas voltadas à remoção dos obstáculos na adoção da gestão ambiental nas MPEs e, também, apoiar a tomada de decisão dos empresários quanto à introdução de políticas ambientais em seus negócios.

\subsection{Panorama da gestão ambiental nas micro e pequenas empresas amazônicas}

Diante do estudo realizado, o panorama da gestão ambiental observado na região estudada ainda é pessimista e assustador. A maioria das MPEs possui pouca ou nenhuma motivação, ou viés ambiental. E considerando a Região Amazônica e sua representatividade quando se discute acerca da preservação dos recursos naturais, os dados desta pesquisa são ainda mais alarmantes. Os principais fatores ligados à gestão ambiental encontrados nesses estudos estão resumidos na Tabela 10 .
TABELA 10 - Principais fatores identificados.

\begin{tabular}{lc}
\hline $\begin{array}{l}\text { Práticas ecologicamente corretas mais adotadas } \\
\text { pelas empresas }\end{array}$ & $\mathbf{\%}$ \\
\hline Economia de energia elétrica & $\mathbf{9 9 , 0 2}$ \\
Economia do consumo de água & $\mathbf{9 2 , 1 6}$ \\
Cumprir as Legislações Ambientais & $\mathbf{9 7 , 0 6}$ \\
Destinação adequada do óleo utilizado & $\mathbf{7 7 , 5 5}$ \\
\hline Principais fatores que fariam ou fazem as empresas & $\mathbf{\%}$ \\
adotarem práticas ecologicamente corretas & $\mathbf{1 0 0 , 0 0}$ \\
\hline $\begin{array}{l}\text { Exigência do mercado consumidor } \\
\text { Adequação à legislação ambiental }\end{array}$ & $\mathbf{1 0 0 , 0 0}$ \\
Não pagamento de multas ambientais & $\mathbf{1 0 0 , 0 0}$ \\
Redução de impostos por boas práticas ambientais & $\mathbf{1 0 0 , 0 0}$ \\
Redução de Custos & $\mathbf{1 0 0 , 0 0}$ \\
\hline $\begin{array}{l}\text { Principais barreiras para a adoção de práticas } \\
\text { ecologicamente corretas pelas empresas }\end{array}$ & $\mathbf{0}$ \\
\hline $\begin{array}{l}\text { Falta de incentivo financeiro ou governamental } \\
\text { Alto custo de implantação } \\
\text { Falta de interesse ou cobrança por parte dos consumi- } \\
\text { adotadas }\end{array}$ & $\mathbf{9 7 , 0 5}$ \\
\hline & $\mathbf{9 5 , 1 0}$ \\
\hline
\end{tabular}

\section{Conclusão}

$\mathrm{O}$ presente trabalho pretendeu dar início à discussão acerca da gestão ambiental em micro e pequenas empresas numa região tão ecológica e ambientalmente visada, que é a Amazônia, apresentando como está o panorama ambiental nessas empresas.

Este é um projeto piloto que obteve dados de pesquisa satisfatórios e possibilitou concluir que, na Região Amazônica, a gestão ambiental ainda é pautada pela busca da redução de custos de produção pelas empresas, e não visando necessariamente 
a preservação do meio ambiente e conservação dos recursos naturais.

As práticas ecologicamente corretas mais difundidas e adotadas pelas empresas da região são as que visam redução de custos pela diminuição do uso de recursos naturais, como água e luz, e o cumprimento da legislação ambiental como forma de evitar multas e embargo das atividades pelos órgãos fiscalizadores.

Os principais fatores que fazem ou fariam as empresas adotarem boas práticas estão, igualmente, relacionadas a questões econômicas, dentre os quais: exigência do mercado consumidor, redução de custos, não pagamentos de multas ambientais, dentre outras. Enquanto que entre as principais barreiras encontradas estão: a falta de incentivo financeiro ou governamental, o alto custo de implantação das práticas e a falta de interesse ou cobrança por parte dos consumidores.

É importante ressaltar que a participação do governo e dos consumidores no processo de adoção da gestão ambiental pelas empresas se mostrou de suma importância, sendo estes apresentados como principais atores que possibilitam uma mudança no panorama atual, exigindo boas práticas para conservação de recursos e proteção do meio ambiente.

Espera-se, com esta pesquisa, alcançar pesquisadores, consumidores e cidadãos que possam de fato prover mudanças nesse panorama assustador. E, ainda, que possam exigir ações e medidas específicas do governo no que diz respeito à gestão ambiental em micro e pequenas empresas.

Algumas limitações encontradas ao longo do processo de realização deste estudo precisam ser apontadas, dentre elas: a fraca adesão dos gestores das empresas no preenchimento do questionário e o grau de generalização dos dados, devido às limitações do processo de amostragem e à dimensão da amostra.

Considerando os resultados obtidos e as limitações apontadas, sugerem-se as seguintes linhas de orientação para pesquisas futuras: alargar o estudo a empresas de outros portes, ramos e regiões; e elaborar propostas de planeamento e gestão ambiental para a localidade, no sentido de incentivar a adoção de práticas ecologicamente corretas por parte das empresas.

\section{Referências}

ABNT - Associação Brasileira de Normas Técnicas. NBR ISO 14001:2015: Sistemas de Gestão Ambiental - Requisitos com orientações para uso. Rio de Janeiro. 2015a.

Anthony, R. N.; Govindarajan, V. Management control systems. Boston: McGraw-Hill/Irwin, 12. ed., 2007.

Aragón-Correa, J. A.; Hurtado-Torres, N.; Sharman, S.; García-Morales, V. J. Environmental strategy and performance in small firms: a resource-based perspective. Journal of Environmental Management, 86(1), 88-103, 2008.

Atanase, A.S.I.; Visan, S. Good practices preceding the implementation of the system of management of environment, on small and medium enterprises. The amfiteatru economic journal, 13, 698-711, 2011.

Barbieri, J. C. Gestão ambiental empresarial: conceitos, modelos e instrumentos. São Paulo: Saraiva, 4. ed., 2016.

Battisti, M.; Perry, M. Walking the talk? Environmental responsibility from the perspective of small-business owners. Corporate Social Responsibility and Environmental Management, 18(3), 172-185, 2011.

Blundel, R.; Monaghan, A.; Thomas, C. SMEs and environmental responsibility: a police perspective. Business Ethics: A European Review, 22(3), 246-262, 2013.

Brammer, S.; Hoejmose, S.; Marchant, K. Environmental management in SMEs in the UK: practices, pressures and perceived benefits. Business Strategy and the Environment, 21(7), 423-434, 2012. 
Brønn, P.S.; Vidaver-Cohen, D. Corporate motives for social initiative: legitimacy, sustainability, or the bottom line? Journal of Business Ethics, 87, 91-109, 2009.

Carlsen, J.; Morrison, A.; Weber, P. Lifestyle oriented small tourism firms. Tourism Recreation Research. 33(3), 255-263, 2008.

Carroll, A. B.; Shabana, K. M. The business case for corporate social responsibility: A review of concepts, research and practice. International Journal of Management Reviews, 12(1), 85-105, 2010.

Cassells, S.; Lewis, K. SMEs and environmental responsibility: do actions reflect attitudes? Corporate Social Responsibility and Environmental Management, 18, 186-199, 2011. doi: $10.1002 /$ csr.269

Cuerva, M. C.; Triguero-Cano, A.; Córcoles, D. Drivers of green and non-green innovation: empirical evidence in Low-Tech SMEs. Journal of Cleaner Production, 68(1), 104-113, 2014.

Del Brío, J. Á.; Junquera, B. A review of the literature on environmental innovation management in SMEs: implications for public policies. Technovation, 23, 939-948, 2003. doi: 10.1016/S0166-4972(02)00036-6

EC - European Commission. Flash Eurobarometer 342: SMEs, Resource Efficiency and Green Markets, 2012. Disponível em: $<$ http://ec.europa.eu/public_opinion/flash/ fl_342_en.pdf $>$. Acesso em: abr. 2018.

Falsarella, O. M.; Jannuzzi, C. A. S. C. Planejamento Estratégico Empresarial e Planejamento de Tecnologia de Informação e Comunicação: uma abordagem utilizando projetos. Gestão \& Produção, 24(3), 610-621, 2017.

Fassin, Y.; Van Rossem, A.; Buelens, M. Small-business owner-managers' perceptions of business ethics and CSR-related concepts. Journal of Bussiness Ethics., 98, 425-453, 2011.

Fernández-Viñe, M. B.; Gómez-Navarro, T.; Capuz-Rizo, S. F. Eco-efficiency in the SMEs of Venezuela: current status and future perspectives. Journal of Cleaner Production, 18(8), 736-746, 2010.

Ferreira, J.C. O Pará e seus Municípios. Belém: J.C.V Ferreira, 2003.
Font, X.; Garay, L.; Jones, S. Sustainability motivations and practices in small tourism enterprises in European protected areas. Journal of Cleaner production, 137, 1439-1448, 2016.

Fuller, T.; Tian, Y. Social and symbolic capital and responsible entrepreneurship: an empirical investigation of SME narratives. Journal of Business Ethics, 67, 287-304, 2006.

Gadenne, D. L.; Kennedy, J.; Mckeiver, C. An empirical study of environmental awareness and practices in SMEs. Journal of Business Ethics, 84(1), 45-63, 2009.

Garay, L.; Font, X. Doing good to do well? Corporate social responsibility reasons, practices and impacts in small and medium accommodation enterprises. International Journal of Hospitality Management., 31(2), 328-336, 2012.

Graafland, J. J. Ecological impacts of the ISO14001 certification of small and medium sized enterprises in Europe and the mediating role of networks. Journal of Cleaner Production, 174, 273-282, 2018.

Gray, C.; Mabey, C. Management development key differences between small and large businesses in Europe. International Small Business Journal, 23(5), 467-485, 2005.

Gunnarsson, K.; Andersson, I.-M.; Rosen, G. Systematic work environment management: experiences from implementation in Swedish small-scale enterprises. Industrial health, 48(2), 185-196, 2010.

Haddock-Fraser, J. E; Tourelle, M. Corporate motivations for environmental sustainable development: exploring the role of consumers in stakeholder engagement. Business Strategy and the Environment, 19, 527-542, 2010. doi: 10.1002/bse.663.

Hahn, T.; Pinkse, J.; Preuss, L.; Figge, F. Tensions in corporate sustainability: towards an integrative framework. Journal of Bussiness. Ethics, 127, 297-316, 2015. doi: 10.1007/s10551-014-2047-5

Heras, I.; Arana, G. Alternative models for environmental management in SMEs: the case of Ekoscan vs. ISO 14001. Journal of Cleaner Production, 18(8), 726-735, 2010.

Hillary, R. Small and medium-sized enterprises and the environment: business imperatives. Sheffield: Greenleaf Publishing, 2000. 
Hillary, R. Environmental management systems and the smaller enterprise. Journal of Cleaner Production, 12(6), 561-569, 2004.

Hofmann, K. H.; Theyel G.; Wood, C. H. Identifying firm capabilities as drivers of environmental management and sustainability practices -evidence from small and medium-sized manufacturers. Business Strategy and the Environment, 21, 530-545, 2012. doi: 10.1002/bse.739

Huang, Z.; Brown, A. An analysis and classification of problems in small business. International Small Business Journal, 18(1), 73-85, 1999.

Hussey, D. M.; Eagan, P. D. Using structural equation modeling to test environmental performance in small and medium-sized manufactures: can SEM help SMEs? Journal of Cleaner Production, 15(4), 303-312, 2007.

Husillos, J.; Alvarez Gil, M. J. A stakeholder-theory approach to environmental disclosures by small and medium enterprises (SMES). Revista de Contabilidad-Spanish Accounting Review., 11(1), 125-156, 2008.

IPEA - Instituto de Pesquisa Econômica Aplicada. Brasil em Desenvolvimento 2010: Estado, Planejamento e Políticas Públicas, 2010. Disponível em: < https://ipea.gov.br/ agencia/images/stories/PDFs/livros/livros/livro _federalis moabrasileira_v08.pdf\#page=25>. Acesso em: jul. 2018.

Iraldo, F.; Testa, F.; Frey, M. Is an environmental management system able to influence environmental and competitive performance? The case of the ecomanagement and audit scheme (EMAS) in the European Union. Journal of Cleaner Production., 17(16), 1444-1452, 2009.

Jansson, J.; Nilsson, J.; Modig, F.; Hed Vall, G. Commitment to sustainability in small and medium-sized enterprises: The influence of strategic orientations and management values. Business Strategy and the Environment, 26(1), 69-83, 2017.

Johannson, L. The challenge of implementing ISO 14001 for small and medium-sized enterprises surviving in the new global jungle. Environmental Quality Management, 7(2), 9-19, 1997.

Jorge, M. L.; Madueño, J. H.; Martínez-Martínez, D.; Sancho, M. P. L. Competitiveness and environmental performance in Spanish small and medium enterprises: is there a direct link? Journal of Cleaner Production, 101, 26-37, 2015.

Klewitz, J.; Hansen, E. G. Sustainability-oriented innovation of SMEs: a systematic review. Journal of Cleaner Production, 65, 57-75, 2014. doi: 10.1016/j.jclepro.2013 .07.017

Leone, N. M. C. P. G. As especificidades das pequenas e médias empresas. Revista de Administração, 34(2), 91-94. 1999.

Lewis, K. V.; Cassels, S.; Roxas, H. SMEs and the potential for a collaborative path to environmental responsibility. Business Strategy and the Environment, 24(8), 750-764. 2014.

Likert, R. A technique for the measurement of attitudes. In: Woodworth, R. S (Ed). Archives in Psychology. New York: The Science Press, p. 1- 55, 1932.

Link, S.; Naveh, E. Standardization and discretion: does the environmental standard ISO 14001 lead to performance benefits? IEEE Transactions on Engineering Management, 53(4), 508-519, 2006.

Martins, P. S.; Escrivão Filho, E.; Nagano, M. S. Fatores contingenciais da gestão ambiental em pequenas e médias empresas. RAM. Revista de Administração Mackenzie, 17(2), 156-179, 2016.

Massoud, M. A.; Fayad, R.; El-Fadel, M.; Kamleh, R. Drivers, barriers and incentives to implementing environmental management systems in the food industry: a case of Lebanon. Journal of Cleaner Production, 18(3), 200-209, 2010.

Mckeiver, C.; Gadenne, D. Environmental management systems in small and medium businesses. International Small Business Journal, 23(5), 513-537, 2005.

Mendonça, F. C.A.; Nahum, C. Panorama de aplicação da norma ISO 14001 no Norte do Brasil. In: Anais do $6^{\circ}$ Congresso Internacional de Tecnologia para o Meio Ambiente. Bento Gonçalves, 10 a 12 de abril de 2018. Disponível em: $<$ https:// siambiental.ucs. br/congresso/anais/trabalhosTecnicos? $\mathrm{ano}=2018>$. Acesso em: 12 de novembro de 2020 .

Miles, M. P.; Munilla, L. S.; Mcclurg, T. The impact of ISO 14000 environmental management standards on small and medium sized enterprises. Journal of Quality Management, 4(1), 111-122, 1999. 
Moore, S. B.; Manring, S.L. Strategy development in small and medium sized enterprises for sustainability and increased value creation. Journal of Cleaner Production, 17, 276-282, 2009. doi: 10.1016/j.jclepro.2008.06.004

Moura, L. A. A. de. Qualidade e gestão ambiental: sugestões para implantação das normas ISO 14.000 nas empresas. São Paulo: Juarez de Oliveira, 2000.

Nordic Council Of Ministers. Environmental Incentives and Nordic SMEs: Impact on Performance and Competitiveness, 2005. Disponível em: <http://www.norden.org/sv/ publikationer/publikationer/2005-543>. Acesso em: abril de 2018.

North, P.; Nurse, A. "War stories": morality, curiosity, enthusiasm and commitment as facilitators of SMEs owner's engagement in low carbon transitions. Geoforum, 52, 32-41, 2014.

OECD - Organization for Economic Co-Operation and Development. Small Business and Environmental Compliance. Disponível em: <www.oecd.org/countries/georgia/ 42072908.pdf. 2007>. Acesso em: abril de 2018.

Perez-Sanchez, D.; Barton, J. R.; Bower, D. Implementing environmental management in SMEs. Corporate Social Responsibility and Environmental Management, 10(2), 67-77, 2003.

Rao, P.; Singh, A. K.; O'castillo, O. L.; Intal, P. S. Jr.; Sajid, A. A metric for corporate environmental indicators... for small and medium enterprises in the Philippines. Business Strategy and the Environment, 18(1), 14-31, 2009.

Redmond, J.; Walker, E.; Wang, C. Issues for small business with waste management. Journal of Environmental Management, 88(2), 275-285, 2008.

Revell, A.; Blackburn, R. The business case for sustainability? An examination of small firms in the UK's construction and restaurant sectors. Business Strategy and the Environment, 16(6), 404-420, 2007.

Revell, A.; Stokes, D.; Chen, H. Small businesses and the environment: turning over a new leaf? Business Strategy and the Environment, 288, 273-288. doi: 10.1002/bse. 2010

Roxas, B.; Coetzer, A. Institutional environment, managerial attitudes and environmental sustainability orientation of small firms. Journal of Business Ethics, 111(4), 461-476,
2012.

Rutherfoord, R.; Blackburn, R. A.; Spence, L. J. Environmental management and the small firm: an international comparison. International Journal of Entrepreneurial Behaviour \& Research, 6(6), 310-325, 2000.

Salzmann, O.; Ionescu-Somers, A.; Steger, U. The business case for corporate sustainability. European Management Journal, 23, 27-36, 2005. doi: 10.1016/j.emj.2004.12.007

Sanches, C. S. Gestão ambiental proativa. Revista de Administração de Empresas, 40(1), 76-87, 2000.

Sandhu, S.; Ozanne, L. K.; Smallman, C.; Cullen, R. Consumer driven corporate environmentalism: fact or fiction? Business Strategy and the Environment, 19, 356-366, 2010. doi:10.1002/bse. 686

Sarkis, J.; Dijkshoorn, J. Relationships between solid waste management performance and environmental practice adoption in Welsh small and medium-sized enterprises (SMEs). International Journal of Production Research, 45(21), 4989-5015, 2007.

Schaper, M. Small firms and environmental management: predictors of green purchasing in western Australian pharmacies. International Small Business Journal, 20(3), 235-251, 2002.

Schleich, J. Barriers to energy efficiency: a comparison across the German commercial and services sector. Ecological Economics, 68(7), 2150-2159, 2009.

Schleich, J.; Gruber, E. Beyond case studies: barriers to energy efficiency in commerce and the services sector. Energy Economics, 30, 449-464, 2008.

SEBRAE - Serviço Brasileiro de Apoio às Micro e Pequenas Empresas. Dieese. Anuário do trabalho na micro e pequena empresa, 2013. Disponível em: <www.sebrae. com.br/Sebrae/Portal\%20Sebrae/Anexos/Anuario \%20 do $\% 20$ Trabalho $\% 20 \mathrm{Na} \% 20$ Micro $\% 20 \mathrm{e} \% 20$ Pequena $\% 20$ Empresa_2013.pdf $>$. Acesso em: dezembro de 2017.

Seráfico, J.; Seráfico, M. A Zona Franca de Manaus e o capitalismo no Brasil. Estudos Avançados, 19(54), 99-113, 2005.

Shaw, G.; Williams, A. M. From lifestyle consumption to lifestyle production: changing patterns of tourism entrepre- 
neurship. In: Thomas, R. (Ed.). Small Firms in Tourism: International Perspectives. Oxford: Pergamon, p. 99-114, 2004.

Shi, H.; Peng, S. Z.; Liu, Y.; Zhong, P. Barriers to the implementation of cleaner production in Chinese SMEs: government, industry and expert stakeholders' perspectives. Journal of Cleaner Production, 16(7), 842-852, 2008.

Silva, D. C. C.; Da Silva, E.; Pires, G. R.; Chaves, M. S. A importância das micro e pequenas empresas paraenses na geração de emprego no estado. Inclusão Social, 9(2), 71-82. 2016.

Silva, M. L. da. Recursos florestais e sua transformação em cultura na cidade: reconstituindo significados. In: SIMONIAN, Ligia. T. Lopes (Org.). Belém do Pará história, cultura e sociedade. Belém: NAEA/UFPA, p. 449-489, 2010.

Simpson, M.; Taylor, N.; Barker, K. Environmental responsibility in SMEs: does it deliver competitive advantage? Business Strategy and the Environment, 13(3), 156-171, 2004.

Searcy, C. Measuring enterprise sustainability. Business Strategy and the Environment 25, 120-133, 2016. doi: 10.1002/bse.1861

Seiffert, M. E. B. ISO 14001 Sistemas de Gestão Ambiental: implantação objetiva e econômica. São Paulo: Atlas, 5. ed., 2017.

SEBRAE. Pequenos negócios abrigam 70\% dos ocupados do setor privado, 2017. Disponível em: $<\mathrm{http}: / / \mathrm{www}$. agenciasebrae.com.br/sites/asn/uf/NA/pequenos-negocios-abrigam-70-dos-ocupados-do-setorprivado,c8c0cb57c3b1e510VgnVCM1000004c00 210aRC RD>. Acesso em: julho de 2018.

Spence, L.; Schmidpeter, R.; Habisch, A. Assessing social capital: small and medium sized enterprises in Germany and the U.K. Journal of Business Ethics, 47, 17-29, 2003.

Studer, S.; Welford, R.; Hills, P. Engaging Hong Kong business in environmental change: drivers and barriers. Business Strategy and the Environment, 15(6), 416-431, 2006.

Tamajón, L. G.; Aulet, X. F. Corporate social responsibility in tourism small and médium enterprises evidence from Europe and Latin America. Tourism Management Perspec- tives, 7, 38-46, 2013.

Terence, A. C. F.; Escrivão, E. Planejamento estratégico na pequena empresa: as particularidades das pequenas empresas no processo estratégico. In: Anais do Encontro Nacional de Engenharia de Produção, Salvador, BA, 17 a 19 de outubro de 2001.

Thompson Jr, A. A.; Strickland II, A. J.; Gamble, J. E. Administração estratégica. Porto Alegre: Editora AMGH, 15. ed., 2008.

Tikul, N. Assessing environmental impact of small and medium ceramic tile manufacturing enterprises in Thailand. Journal of Manufacturing Systems, 33(1), 1-6, 2014.

Tilley, F. The gap between the environmental attitudes and the environmental behaviour of small firms. Business Strategy and the Environment, 8(4), 238-248, 1999.

Trianni, A.; Cagno, E.; Worrell, E. Innovation and adoption of energy efficient technologies: an exploratory analysis of Italian primary metal manufacturing SMEs. Energy Policy, 61, 430-440, 2013.

Torrès, O.; Julien, P. A. Specificity and denaturing of small business. International Small Business Journal, 23(4), 355-377, 2005.

Witjes, S.; Vermeulen, W. J.; Cramer, J. M. Exploring corporate sustainability integration into business activities. Experiences from 18 small and medium sized enterprises in the Netherlands. Journal of Cleaner Production, 153, 528-538, 2017.

Wong, C.W; Lai, K-H; Shang, K-C; Lu, C-S. Uncovering the value of green advertising for environmental management practices. Business Strategy and the Environment, 23, 117-130, 2014. doi: 10.1002/bse.1776

Zeng, S. X.; Meng, X. H.; Zeng, R. C.; Tam, C. M.; Tam, V. W. Y.; Jin, T. How environmental management driving forces affect environmental and economic performance of SMEs: a study in the Northern China district. Journal of Cleaner Production, 19(13), 1426-1437, 2011.

Zorpas, A. Environmental management systems as sustainable tools in the way of life for the SMEs and VSMEs. Bioresource technology, 10(6), 1544-1557, 2010. 